\title{
Diagnostic reliability of pediatric appendicitis score, ultrasound and low-dose computed tomography scan in children with suspected acute appendicitis
}

\author{
This article was published in the following Dove Press journal: \\ Therapeutics and Clinical Risk Management \\ 6 July 2017 \\ Number of times this article has been viewed
}

\author{
Ashraf Othman Sayed' \\ Nancy Selim Zeidan ${ }^{2}$ \\ Dalia Monir Fahmy ${ }^{3}$ \\ Hossam A lbrahim ${ }^{4}$ \\ 'Department of Pediatrics, Children \\ and Women's University Hospital, \\ Minia University, El-Minya, Egypt; \\ ${ }^{2}$ Department of Pediatrics, Faculty \\ of Medicine, Cairo University, \\ Cairo, Egypt; ${ }^{3}$ Department of \\ Diagnostic Radiology, Mansoura \\ University Hospital, Faculty of \\ Medicine, Mansoura University, \\ Mansoura, Egypt; ${ }^{4}$ Department of \\ Surgery, Faculty of Medicine, Cairo \\ University, Cairo, Egypt
}

\begin{abstract}
Background: Diagnosis of appendicitis in children is clinically challenging. Computed tomography $(\mathrm{CT})$ is the gold standard for diagnosis; however, radiation exposure early in life is a concern with this technique. Therefore, in this study, we aimed to evaluate the diagnostic reliability of low-dose CT, pediatric appendicitis score (PAS), and abdominal ultrasound (US) in children with acute appendicitis, to reach a safe diagnosis.
\end{abstract}

Patients and methods: This retrospective study was conducted on 140 children who were admitted with clinically suspected acute appendicitis (45 with positive appendicitis and 95 children with negative appendicitis). Low-dose CT was performed, and PAS was retrospectively calculated for all subjects. US was initially performed for 38 subjects. All results were compared with the final diagnosis reached by an operative, histopathological analysis and follow-up.

Results: Low-dose CT showed a sensitivity, specificity, and accuracy of $97.8 \%, 100 \%$, and $99.3 \%$, respectively. At a cutoff value $\geq 5$, PAS showed a sensitivity, specificity, and accuracy of $95 \%, 84 \%$, and $89 \%$, respectively. Abdominal US examination showed sensitivity, specificity, and accuracy of $55.6 \%, 85 \%$, and $71 \%$, respectively. Implementing Poortman's model resulted in higher accuracy (92\%) of US. There was a significant difference in accuracy between a low-dose CT and PAS on one side and between Poortman's model and US examination on the other side. A diagnostic scheme was suggested using PAS as the excluding tool (PAS $\leq 2$ send home and $\geq 7$ send directly to operation) followed by US examination and reserving low-dose CT for inconclusive cases. This scheme would eliminate the use of CT for at least $33.7 \%$ and in 7 cases who had initial US examination.

Conclusion: Although CT remains the most accurate and less operator-dependent diagnostic tool for pediatric appendicitis, the radiation hazards could however be minimized using PAS as an excluding tool and US as the primary imaging modality followed by low-dose CT for inconclusive cases only.

Keywords: acute appendicitis, children, pediatric appendicitis scoring system, PAS, computed tomography, CT, ultrasound, US

\section{Introduction}

Acute appendicitis remains the most common acute surgical condition in children and a major cause of morbidity; appendectomy is still the mainstay of treatment. ${ }^{1}$ Delayed intervention leads to dramatic complications such as perforation and abscess formation while rushing to surgery is associated with a high negative appendectomy 
of up to $15 \%-30 \% .^{2}$ Diagnosis of appendicitis in children is challenging, not only because there are so many other nonsurgical conditions that mimic appendicitis, ${ }^{1}$ but also because there are difficulties in communication and examination. ${ }^{3}$

In many hospitals, including ours, pelvic-abdominal computed tomography (CT) is considered the gold standard diagnostic tool for the diagnosis of appendicitis in children owing to its high sensitivity and specificity. ${ }^{4} \mathrm{CT}$ scan has certainly saved a significant number of patients from undergoing unnecessary surgeries but has also caused unnecessary radiation exposure in them. Recent studies have reported that the risk of radiation exposure early in life is up to $25 \mathrm{mSv}$ per study, which has raised concern regarding the increased incidence of cancer in pediatric patients. ${ }^{5,6}$ Another study predicted that approximately 29,000 future cancer cases will be linked to CTs performed in the year 2007, with the largest proportion coming from pelvic-abdominal scans. ${ }^{7}$ Therefore, in order to decrease radiation exposure, several protocols of modified technical parameters (tube voltage, tube current, and slice thickness) have been implemented. ${ }^{8}$

Graded compression abdominal ultrasound (US) is a widespread bedside examination tool which costs less than $\mathrm{CT}$ and poses no radiation hazards but has low sensitivity and is operator-dependent. Pediatric appendicitis score (PAS) is a commonly cited appendicitis clinical scoring system that was developed specifically for children by Samuel. ${ }^{9}$ To our knowledge, there are no previously published studies that investigated PAS performance in comparison to abdominal US and low-dose CT in our region.

Therefore, we aimed to evaluate the diagnostic reliability of low-dose CT, PAS, and abdominal US examination in children with suspected acute appendicitis, in order to reach a safe diagnosis with less radiation hazard.

\section{Subjects and methods Design and study population}

This is a retrospective cross-sectional study conducted over 18 months from March 2015 to September 2016 at a major urban institution. Ethical approval from the Ethic Committee of Dar Al-Shifa Hospital was obtained for this study. The committee waived the need for an informed patient consent (from parent or guardian) owing to the retrospective nature of the research and as it did not seem to jeopardize patient confidentiality, privacy, or safety.

\section{Inclusion criteria}

All children of both sexes between the age of 4 and 18 years who were admitted with clinically suspected acute appendicitis were included in this study.

\section{Exclusion criteria}

Children aged below 4 or above 18 years at the time of operation, or with incomplete medical records, or those who underwent appendectomy incidentally, or with chronic abdominal pain were excluded from this study.

\section{Result comparison with final diagnosis}

The PAS and imaging (CT and US) results were compared with the final diagnosis reached by surgery and histopathological analysis or by follow up.

\section{Clinical and laboratory assessment}

The following data were collected from the automated and nonautomated medical records in the hospital: age, gender, duration of abdominal symptoms (days), and weight (kg).

PAS values were calculated retrospectively for each patient according to the original PAS definition. ${ }^{9}$ The 8 components of PAS are as follows: fever, anorexia, nausea/ vomiting, migration of the pain to the right lower quadrant (RLQ), tenderness on light palpation of RLQ, cough/ percussion/heel tapping tenderness at the RLQ, leukocytosis, and polymorphonuclear neutrophilia; all components of PAS were scored 1 point, except, right quadrant tenderness and cough/percussion/heel tapping tenderness were scored 2 points (Table 1). Clinical assessment was performed by licensed pediatricians in the emergency room upon the initial admission of the patients.

\section{Radiological imaging Computed tomography}

All subjects $(\mathrm{n}=140)$ included in this study had CT examination of the abdomen and pelvis, which was performed after fasting for at least 4 hours prior to scanning. Our protocol included intake of $1,000 \mathrm{~mL}$ of oral contrast solution (nonionic) over a period of $90 \mathrm{~min}$, followed by pre- and postcontrast phase (venous). However, in certain circumstances, such as severe vomiting or urgent cases that were planned for

Table I Components of pediatric appendicitis score

\begin{tabular}{ll}
\hline Signs/symptoms & $\begin{array}{l}\text { Point } \\
\text { value }\end{array}$ \\
\hline Nausea/emesis & $\mathrm{I}$ \\
Anorexia & $\mathrm{I}$ \\
Migration of pain to RLQ & $\mathrm{I}$ \\
Low-grade fever $\left(\geq 38.0^{\circ} \mathrm{C}\right)$ & $\mathrm{I}$ \\
RLQ tenderness on light palpation & 2 \\
Cough/percussion/heel tapping tenderness at RLQ & 2 \\
Leukocytosis $\left(>10,000 / \mathrm{mm}^{3}\right)$ & $\mathrm{I}$ \\
Left shift $(>75 \%$ neutrophilia) & $\mathrm{I}$ \\
Total & $\mathrm{I} 0$ \\
\hline
\end{tabular}

Abbreviation: RLQ, right lower quadrant. 
operation soon after CT scan, oral intakes of contrast were eliminated from our protocol.

CT scan was performed using a Siemens SOMATOM defined Flash dual source 128 multi-detector scanner (Siemens Medical Solutions, Forchheim, Germany): tube voltage, $100 \mathrm{kVp}$; tube current, 87/190 MA; slice thickness, $6 \mathrm{~mm}$; and $40 \mathrm{~mL}$ Xenetix ${ }^{\circledR}$ (Guerbet, Gorinchem, the Netherlands) at $4 \mathrm{~m} / \mathrm{s}$. Postcontrast scan was performed $60 \mathrm{~s}$ after intravenous injection of Xenetix 350. The low-dose technique was implemented and a size-specific dose estimate (SSDE), an approximation of absorbed dose incorporating patient diameter, and effective dose (ED) was calculated for each scan.

\section{Image analysis}

$\mathrm{CT}$ images were reviewed by a senior radiologist (DF) with 15 years' experience in abdominal imaging, who was blinded to the clinical findings and laboratory results. The criteria for diagnosis of acute appendicitis included the following: swollen appendix (outer diameter exceeding $6 \mathrm{~mm}$ ) with or without fecolith, enhanced walls, and peri-appendiceal strands. The signs of perforation included the following: free intra-peritoneal air and excess peri-appendiceal, pelvic, or abdominal fluid. CT findings were interpreted as negative if an appendix was not visualized, had an outer diameter of less than $6 \mathrm{~mm}$, and had absence of peri-appendiceal strands.

\section{Ultrasound examination}

All US examinations were performed using a curved 3.5-5.0 MHz array and a linear $6 \mathrm{MHz}$ array (ultrasound machine GE volusone E8 and E10). The criteria for diagnosis of acute appendicitis were as follows: local transducer tenderness, noncompressible appendix, a thickened appendix (diameter greater than $6 \mathrm{~mm}$ ), presence of an appendicoliths, peri-appendiceal fat infiltration, and free fluid adjacent to the appendix. In addition to a routine abdominal and pelvic US, all ultrasound examinations were performed in our institution by licensed general radiologists with at least 5 years' experience.

\section{Operative and histopathological analysis}

Surgery was performed by a consultant general surgeon with more than 20 years' of experience. The existence of polymorphonuclear leukocytes, lymphocytes, or plasma cells in appendiceal biopsy was considered positive for appendicitis.

Negative appendectomy was defined as, 1) an operation with a preoperative diagnosis of appendicitis, and 2) absence or minimal acute inflammatory cells in the case of appendectomy, or normal appearance of the appendix.

\section{Follow-up}

Patients who had a stable clinical condition and with a negative CT scan for appendicitis were sent home and received a follow-up phone call after 1 week to assess their condition and cessation of symptoms. Patients, who had other diseases that caused acute abdominal pain other than appendicitis, discovered by imaging and laboratory tests, were treated according to their condition, as usual.

\section{Statistical analysis}

IBM SPSS software package (Statistical Package for Social Sciences, version 20 for Windows) was used to analyze data. A 1-sample Kolmogorov-Smirnov test was used to assess whether the data were normally distributed. Continuous variables were presented as mean \pm standard deviation and data were compared using an unpaired $t$-test. Categorical variables were expressed as numbers and percentages and analyzed for comparisons using chi-square test.

For evaluating the predictive value of PAS in the diagnosis of acute appendicitis, the sensitivity, specificity, positive predictive value (PPV), negative predictive value (NPV), and accuracy were calculated. In addition, receiver operating characteristic (ROC) curves were analyzed for the overall PAS performance. At the $5 \%$ level of significance, $P$-value less than 0.05 was considered significant in all statistical tests.

\section{Results}

A total of 140 patients were included in this study; 45 patients (positive appendicitis group) had surgery followed by histopathological analysis that confirmed acute appendicitis. None had a negative appendectomy. The remaining 95 patients (negative appendicitis group) had diseases other than appendicitis that were revealed by CT scan and clinical follow-up; none of these patients showed any complications related to a missed diagnosis of appendicitis (Table 2).

Table 2 The final diagnosis in the negative appendicitis group $(n=95)$

\begin{tabular}{ll}
\hline Diagnosis & $\begin{array}{l}\text { Number } \\
\text { of cases }\end{array}$ \\
\hline Mesenteric lymphadenitis & 42 \\
Ovarian cyst & 18 \\
Colitis & 25 \\
Enteritis & 4 \\
Crohn's & $\mathrm{I}$ \\
Intestinal obstruction & $\mathrm{I}$ \\
Diverticulitis & $\mathrm{I}$ \\
Acute paniculitis & $\mathrm{I}$ \\
Gastritis & $\mathrm{I}$ \\
Ureteric stone & $\mathrm{I}$ \\
Total & 95 \\
\hline
\end{tabular}


Table 3 Demographic and clinical characteristics of all study patients $(n=\mid 40)^{a}$

\begin{tabular}{|c|c|c|c|}
\hline & $\begin{array}{l}\text { Appendicitis } \\
(n=45)\end{array}$ & $\begin{array}{l}\text { No appendicitis } \\
(n=95)\end{array}$ & $P$-value \\
\hline Age (years) & $13.1 \pm 4$ & $13.2 \pm 3.9$ & $\begin{array}{l}P=0.885^{b} \\
t \text {-value }=-0.144\end{array}$ \\
\hline \multicolumn{4}{|l|}{ Gender } \\
\hline Male & 27 (60\%) & 50 (54\%) & $\chi^{2}=0.6699$ \\
\hline Female & I 8 (40\%) & 45 (46\%) & $P=0.413^{c}$ \\
\hline Weight (kg) & $45.65 \pm 18.2$ & $47.2 \pm 19$ & $\begin{array}{l}t \text {-value }=0.586 \\
P=0.559^{\circ}\end{array}$ \\
\hline $\begin{array}{l}\text { Symptoms } \\
\text { duration (days) }\end{array}$ & $1.85 \pm 0.56$ & $2.0 \pm 1.11$ & $\begin{array}{l}P=0.210^{\mathrm{b}} \\
t \text {-value }=1.258\end{array}$ \\
\hline $\begin{array}{l}\text { Pediatric } \\
\text { appendicitis score }\end{array}$ & $5.34 \pm \mathrm{I} .15$ & $2.48 \pm I .1 I$ & $\begin{array}{l}t \text {-value }=-17.947 \\
P<0.00 I^{b}\end{array}$ \\
\hline
\end{tabular}

Notes: ${ }^{a}$ Continuous variables are presented as mean \pm standard deviation; categorical variables as numbers with percentages; 'Unpaired $t$-test, 'chi-square test.

Out of 140 patients included in this study, 77 were males and 63 were females. No significant difference was found between the positive and negative appendicitis groups regarding patients' gender or weight. Patients' ages ranged from 4 to 16 years and their mean age was ( $11 \pm 0.67$ years). Table 3 summarizes the demographic data.
All patients $(n=140)$ included in this study underwent CT scan; it was the initial imaging in 102 patients and subsequently US imaging was performed for the remaining 38 patients. Forty-four patients had radiological findings coping with acute appendicitis in CT examination, which was correlated with the histopathological findings: 16 showed dense fecolith; perforation was noted in 7 (3 of them were associated with fecolith as shown in Figure 1); and 1 was associated with an acute abscess. The most common location of the appendix with inflammation was pelvic region (19 cases, 42\%) followed by retrocecal (16 cases, 36\%). Table 4 summarizes radiological signs of patients.

Only 1 patient was misdiagnosed by CT as having a normal appendix with a probably complicated right ovarian cyst. As this patient had persistent pain and tenderness in right iliac fossa, she was referred to laparoscopic surgery that revealed mild inflammation in her appendix with right corpus luteum cyst.

CT examination showed a sensitivity of $97.8 \%(95 \%$ confidence interval $[\mathrm{CI}]=88.2 \%-99.9 \%$ ), specificity of $100 \%(95 \% \mathrm{CI}=96.2 \%-100 \%)$, PPV of $100 \%$, NPV of $98.7 \%$ $(95 \% \mathrm{CI}=93.2 \%-99.9 \%)$, and an accuracy of $99.3 \%$.
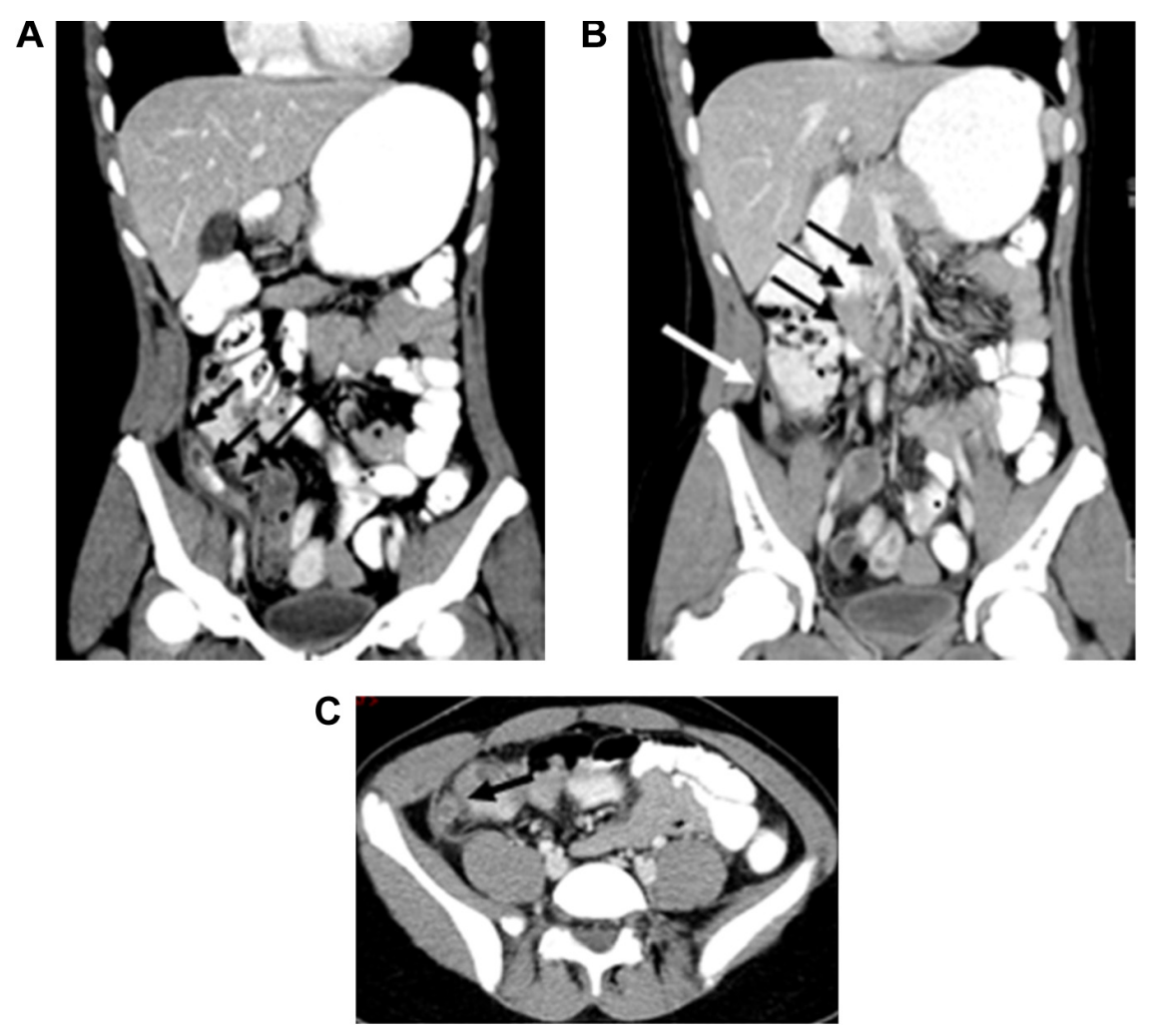

Figure I Preoperative postcontrast abdominal-pelvic CT scan of a child aged 14 years, who presented with abdominal pain and vomiting (PAS score $=5$ ). Notes: (A and B) Coronal reformatted images showed distended appendix with thick edematous walls, diameter $15 \mathrm{~mm}$ (long black arrow in A), which contains fecolith (short double black arrows in A). A tiny focus of air near its tip denoting contained perforation (white arrow in B); multiple associated regional and mesenteric lymph nodes (black arrows in B). (C) Axial image showed distended appendix with thick edematous walls surrounded by peri-appendiceal fat stranding, and mild peri-appendiceal fluid (black arrow in C).

Abbreviations: PAS, pediatric appendicitis score; CT, computed tomography. 
Table 4 Computed tomography (CT) findings in positive appendicitis cases

\begin{tabular}{ll}
\hline CT findings & Patients (n) \\
\hline Edematous wall & 44 \\
Perforation & 8 \\
Fecolith & 16 \\
Fecolith associated with perforation & 3 \\
Enlarged regional and mesenteric lymph nodes & 24 \\
Abscess & 1 \\
According to the appendix location & 19 \\
Pelvic & 16 \\
Retrocecal & 2 \\
Subhepatic & 7 \\
Anterior & \\
\hline
\end{tabular}

The mean dose-length product was $150 \mathrm{mGy} / \mathrm{cm}$ (ranging from 115 to $200 \mathrm{mGy} / \mathrm{cm}$ ) and mean effective dose of radiation was $3.1 \mathrm{mSv}$ (ranging from 2.2 to $3.4 \mathrm{mSv}$ ).

The abdominal US examination was the initial imaging in 38 patients; it gave true positive results in 10 cases $(26.3 \%)$, true negative in 17 cases $(44.7 \%)$, false negative (missed appendicitis) in 8 cases (21\%), and false positive (negative appendectomy) in 3 cases $(7.9 \%)$. It showed a sensitivity of $55.6 \%(95 \% \mathrm{CI}=30.8 \%-78.5 \%)$, specificity of $85 \%(95 \% \mathrm{CI}=62.1 \%-96.8 \%)$, PPV of $76.9 \%(95 \%$ $\mathrm{CI}=52 \%-91 \%), \mathrm{NPV}$ of $68 \%(95 \% \mathrm{CI}=55.1 \%-78.6 \%)$, and an accuracy of $71 \%$. In 2 patients (who were negative for appendicitis), abdominal US examination revealed right ovarian cysts $3 \mathrm{~cm}$ and $3.5 \mathrm{~cm}$ in diameter, respectively.

Following Poortman's model ${ }^{10}$ (which consisted of combination of US as the primary examination followed by CT in nondiagnostic US examination) yielded a sensitivity of $100 \%(95 \% \mathrm{CI}=81.5 \%-100 \%)$, specificity of $85 \%(95 \%$ $\mathrm{CI}=62.1 \%-94.5 \%)$, PPV of $85.7 \%$ (95\% CI $=67.9 \%-78.6 \%)$, an accuracy of $92 \%$, negative appendectomy rate of $7.9 \%$, and no missed positive appendicitis cases. It alone would have avoided the use of CT in 13/38 cases.

There was a significant difference between the PAS in positive and negative appendicitis groups $(P<0.001)$. In this study, PAS score $\geq 5$ was found to be the best cutoff point compatible with acute appendicitis; it resulted in a sensitivity of $95 \%$ ( $95 \%$ CI $=29 \%-98 \%$ ), specificity of $84 \%$ (95\% CI $=76 \%-90 \%)$, PPV of $82 \%(95 \% \mathrm{CI}=73 \%-89 \%)$, NPV of $82 \%(95 \%$ CI $=73 \%-89 \%)$, and accuracy of $89 \%$ (as shown in Figure 2). Further analysis of PAS showed that it is more useful as an exclusive tool; PAS $\geq 2$ showed the highest sensitivity of $97.8 \%$ ( $95 \%$ CI $=88.2 \%-99.9 \%$ ) with only a single false negative case (missed appendicitis), whereas using higher cutoff value (PAS $\geq 7$ ) showed the highest specificity $97.9 \%(95 \% \mathrm{CI}=2.6 \%-99.7 \%)$ with only 2 cases of negative appendectomy (Table 5).

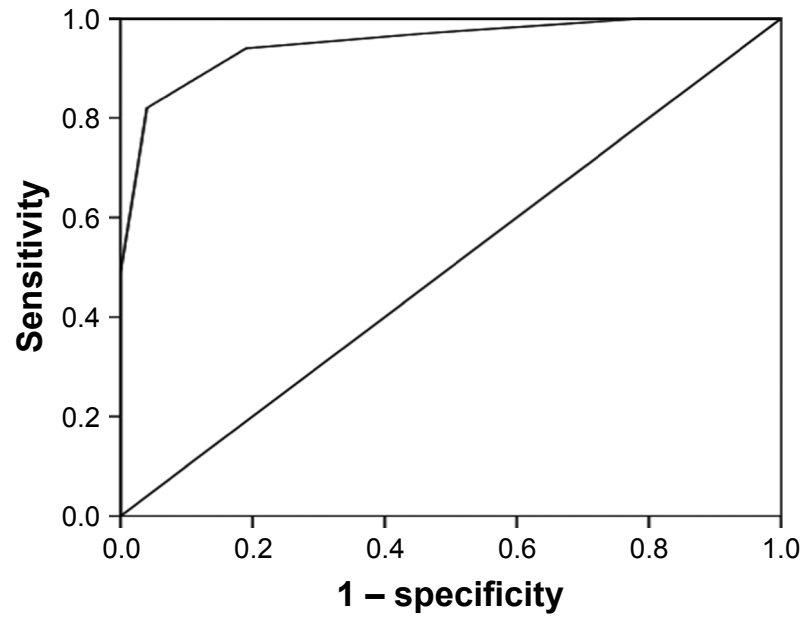

Figure 2 Receiver operating characteristic curve for the performance of pediatric appendicitis score.

Note: Area under curve (95\% confidence interval) $=0.951$ (0.923-0.979).

On comparing low-dose CT, US, Poortman's model, and PAS (using a cutoff value $\geq 5$ ), low-dose CT showed the highest accuracy, whereas US showed the lowest (Table 6).

On one side, there was a significant difference in accuracy between low-dose CT scans and PAS $(P<0.001)$, and on the other side, there was a significant difference between Poortman's model and US $(P<0.02)$.

Finally, we propose a diagnostic scheme that depends on the clinical score (PAS) as an initial diagnostic tool followed by US examination (if PAS is in the range of 3-6), preserving low-dose CT as the last step for cases with inconclusive US findings (as shown in Figure 3). Following this scheme would have eliminated the use of CT for at least 47/140 (33.6\%) patients who had PAS $\leq 2$ or $\geq 7$. Unfortunately, not all patients included in this study had US examination; however, following this scheme for the remaining 38 patients who had initial US examination would have avoided the use of CT in 7 cases (PAS 3-6 and positive US findings).

\section{Discussion}

Owing to its high diagnostic accuracy, $\mathrm{CT}$ is utilized widely in the management of appendicitis, but this trend is accompanied by an increased radiation exposure and long-term

Table 5 Sensitivity and specificity of PAS values (using 3 different cutoff points) in all subjects ( $n=\mid 40$ ), according to final diagnosis

\begin{tabular}{llll}
\hline PAS cutoff & Sensitivity & Specificity & Accuracy \\
\hline PAS $\geq 2$ & $97.8 \%$ & $26.3 \%$ & $49.3 \%$ \\
& $(95 \%$ Cl: 88.2-99.9) & $(95 \%$ Cl: 17.8-36.4) & \\
PAS $\geq 5$ & $95 \%$ & $84 \%$ & $89 \%$ \\
& $(95 \%$ Cl: $29-98)$ & $(95 \%$ Cl: 76-90) & \\
PAS $\geq 7$ & $42.2 \%$ & $97.9 \%$ & $73 \%$ \\
& $(95 \%$ Cl: $27.7-57.9)$ & $(95 \%$ Cl: 92.6-99.7) & \\
\hline
\end{tabular}

Abbreviations: PAS, pediatric appendicitis score; $\mathrm{Cl}$, confidence interval. 
Table 6 Comparison of performance between CT, US, Poortman's model, and PAS

\begin{tabular}{lllll}
\hline & $\begin{array}{l}\text { PAS } \geq \mathbf{5} \\
(\mathbf{n}=\mathbf{1 4 0 )}\end{array}$ & $\begin{array}{l}\mathbf{C T} \\
(\mathbf{n}=\mathbf{1 4 0 )}\end{array}$ & $\begin{array}{l}\text { US } \\
(\mathbf{n}=\mathbf{3 8})\end{array}$ & $\begin{array}{l}\text { Poortman's model } \\
(\mathbf{n}=\mathbf{3 8})\end{array}$ \\
\hline Sensitivity & $95 \%$ & $97.8 \%$ & $55.6 \%$ & $100 \%$ \\
Specificity & $84 \%$ & $100 \%$ & $85 \%$ & $85 \%$ \\
Positive predictive value & $82 \%$ & $100 \%$ & $76.9 \%$ & $85.7 \%$ \\
Negative predictive value & $82 \%$ & $98.7 \%$ & $68 \%$ & $100 \%$ \\
Accuracy & $89 \%$ & $99.3 \%$ & $71 \%$ & $92 \%$ \\
$\begin{array}{l}\text { Negative appendectomy } \\
\text { (false positive) }\end{array}$ & $4(2.8 \%)$ & 0 & $3(7.9 \%)$ & $3(7.9 \%)$ \\
$\begin{array}{l}\text { Missed appendicitis } \\
\text { (false negative) }\end{array}$ & $9(6.4 \%)$ & $1(0.7 \%)$ & $8(21 \%)$ & 0 \\
\hline
\end{tabular}

Abbreviations: CT, computed tomography; PAS, pediatric appendicitis score; US, ultrasound.

cancer risks. ${ }^{4}$ Two main pathways have been suggested to decrease these hazards and maintain high diagnostic accuracy; first to decrease the radiation dose by implementing a low-dose protocol in pediatric CT (image gently), and second, to decrease the utilization of CT by using a clinical score and US examination either alone or prior to CT, which can be performed in case of doubt (this decision should be made wisely).

In the current study, a low-dose protocol was used for all patients with a mean radiation dose of $3.1 \mathrm{mSv}$. Although the low-dose technique resulted in reduced quality of images it did not affect the diagnostic accuracy. CT examination showed a sensitivity of $97.8 \%$, specificity of $100 \%$, and an accuracy of $99.3 \%$. These results agree with other studies ${ }^{11-14}$ in which the authors have reported no significant difference in sensitivity, specificity, negative appendectomy, or missed appendicitis rate between low-dose protocol and regular CT scan.

In the current study, graded compression US was performed for a relatively small group of patients (38 cases). It

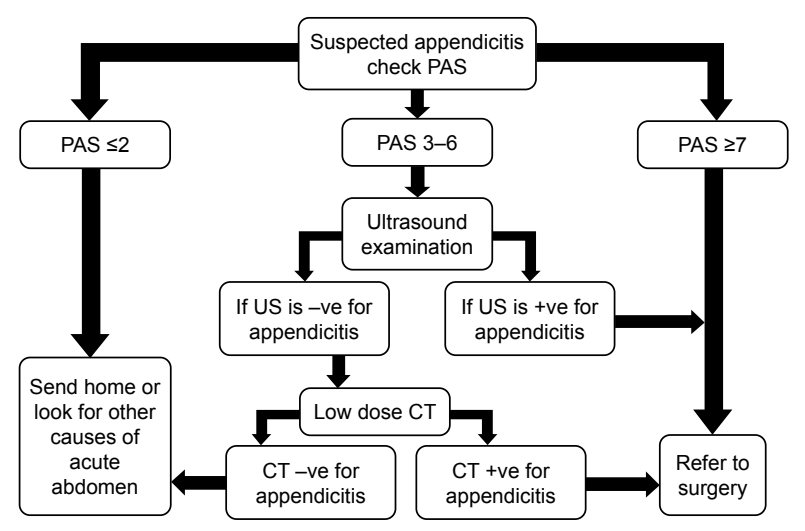

Figure 3 A suggested scheme for the diagnosis of appendicitis using PAS, US, and low-dose CT scan.

Abbreviations: PAS, pediatric appendicitis score; US, ultrasound; CT, computed tomography; -ve, negative; +ve, positive. had less sensitivity (55.6\%), specificity (85\%), and accuracy (71\%) compared to $\mathrm{CT}$, which could be attributed to 2 main reasons. First, all sonographic examinations were performed in our institution by a general radiologist, not by a pediatric sonologist; second, owing to the high percentage of a retrocecal position of the appendix (36\%) which hindered its detection by the US.

Poortman et $\mathrm{al}^{10}$ suggested a diagnostic model for appendicitis that included graded compression US as the initial imaging modality followed by CT only in nondiagnostic US examination. Applying this model to a relatively small group of patients in the current study, it was found that primary US examination dramatically improved the sensitivity (100\%), specificity (85\%), and accuracy (92\%), and yielded a negative appendectomy rate of $8 \%$ and no missed appendicitis. These results are similar to those in the studies of Poortman et $\mathrm{al}^{10}$ (sensitivity of $100 \%$, specificity of $86 \%$, and negative appendectomy $8 \%$ ), Ramarajan et $\mathrm{al}^{15}$ (sensitivity of $99 \%$, specificity of $91 \%$, and negative appendectomy $7 \%$ ), and Thirumoorthi et $\mathrm{al}^{16}$ (sensitivity of $94.2 \%$, specificity of $97.5 \%$, negative appendectomy $1.8 \%$, and missed appendicitis $0 \%$ ).

One study reported an increasing trend of using US as the first imaging tool (about 69\% instead of 32.6\%) during the period from 2008 through 2013, whereas the use of CT was decreased. ${ }^{18}$ In the current study, US examination was the initial imaging modality in $27 \%$ of the cases suspected to have appendicitis. In contradiction to other studies which reported utilization of preoperative $\mathrm{CT}$ in about $40 \%$ of the cases, ${ }^{17-19}$ in our study, all patients undergoing appendectomy had received a preoperative CT, even if the patient had a US diagnosis of appendicitis. The reasons behind this could be that surgeons in our region have less trust in US results as compared to CT, which has higher sensitivity, specificity, and is indeed less operator-dependent. Although CT is more 
expensive than US, it is still less expensive than the cost of negative appendectomy or managing complications such as a perforated appendix. Furthermore, physicians find difficulty in convincing some parents of the diagnosis of acute appendicitis based only on clinical and US findings; they believe that CT scan can be less harmful than doing unnecessary appendectomy with possible surgical complications.

The main drawback of US is that it is highly operatordependent and its accuracy is affected dramatically by the examiner's own experience. That is why CT scan utilization is higher in general hospitals (including our hospital) when compared to specialized pediatric hospitals. This is in agreement with Kotagal et $\mathrm{a}^{17}$ who noted 8 times higher use of $\mathrm{CT}$ in nonpediatric hospitals.

Appendicitis scoring systems have been developed as a diagnostic tool to improve the decision-making process in patients with suspected acute appendicitis. ${ }^{20}$ In the current study, PAS score $\geq 5$ was found to be the best cutoff point compatible with acute appendicitis, with a sensitivity of $95 \%$, specificity of $84 \%$, and an accuracy of $89 \%$. However, there was still a significant difference in accuracy between lowdose CT and PAS. In this study, it has been found that relying on a single PAS cutoff value would result in a negative appendectomy in 4 cases (2.8\%) and missed appendicitis in 9 cases (6.4\%). Other studies suggested a modified pathway utilizing both PAS and imaging; Lourenco et $\mathrm{al}^{21}$ suggested that patients with a PAS of 1 to 3 could be discharged without further imaging examination, patients who had a PAS of 4 to 6 would require further imaging examination, and those who had a PAS of 7 to 10 required surgical consultation without imaging examination. Similarly, Goldman et al ${ }^{22}$ reported that a score of 7 or greater is valid for the diagnosis of appendicitis and a score of 2 or under is valid for the exclusion of appendicitis; and Zúñiga et $\mathrm{a}^{23}$ found that at PAS of $\leq 3$ no patients were diagnosed with acute appendicitis, and if all patients with PAS of 8 or higher were operated on, there was a $5 \%$ rate of negative appendectomy.

Similarly, in the current study, we found that using PAS $\leq 2$ to exclude the diagnosis of appendicitis would have led to missing only 1 case $(0.7 \%)$, whereas using PAS $\geq 7$ as an indication for surgery would have led to negative appendectomy in 2 cases (1.4\%); the rest of the patients having PAS between 3 and 6 were in need of further imaging studies. Accordingly, we have proposed a diagnostic scheme for acute appendicitis (Figure 3) that combines three diagnostic tools (PAS, US, and low-dose CT). Obviously, following this pathway will result in fewer cases of missed appendicitis or negative appendectomy as compared to using US or PAS alone, whilst also lowering CT utilization. We found that following this scheme would have eliminated the use of CT for at least $33.6 \%$ (47/140) of the patients who had PAS $\leq 2$ or $\geq 7$, and in 7 patients who had initial US examination before $\mathrm{CT}$. In contrast, these results disagree with Srinivasan et al ${ }^{24}$ who found little benefit in performing $\mathrm{CT}$ for patients with PAS $<6$ and negative US findings. Again, this could be attributed to the fact that our study was conducted in a general hospital and not in a tertiary pediatric center, and because US was performed by general radiologists and not pediatric sonologists. However, Rezak et $\mathrm{al}^{25}$ reported about $27 \%$ theoretical decrease in CT utilization in children with suspected appendicitis with modified Alvarado score (5-7).

In the current study, PAS that equals 5 or more was found to be the best cutoff value for diagnosis of appendicitis; however, this value is less than that found by several other studies including Samuel ${ }^{9}$ who created this score system back in 2002. This difference could be attributed to inherited difference between prospective and retrospective study. In the current study, examination of patients was performed by pediatricians and not by pediatric surgeons who could have underestimated the clinical signs. Other factors related to local population habits, such as giving children several analgesics and antipyretics without medical prescription, which could mask clinical signs. Another factor is difficulty in communication, as our hospital serves patients coming from multiple nationalities with different languages. To our knowledge, this is the first study to assess the validity of PAS as a diagnostic tool for pediatric appendicitis in our region; further large prospective multicenter study is recommended to clarify more its diagnostic value.

There are some limitations in this study. First, the overall low number of cases included and the even fewer cases that had US examinations. As the number of patients who had US examination was less than those who had CT examination, we were not able to apply Poortman's model and our final diagnostic scheme for all cases. Second, all cases had low-dose CT examination performed, and we were not able to compare between low and ordinary dose $\mathrm{CT}$ in order to get true measurements of degree of dose reduction. Finally, the retrospective nature of this study hindered our ability to assess the feasibility of the suggested diagnostic scheme on daily work instead of assumption.

\section{Conclusion}

Although CT remains the most accurate and less operatordependent diagnostic tool for pediatric appendicitis, radiation hazards could be minimized using PAS as an excluding tool 
and US as primary imaging modality, followed by low-dose CT for inconclusive cases only.

\section{Disclosure}

The authors report no conflicts of interest in this work.

\section{References}

1. Aiken JJ, Oldham KT. Nelson Textbook of Pediatrics. 20th edition, Philadelphia, Elsevier publisher; 2016:1887-1894.

2. Lembcke B. Ultrasonography for acute appendicitis - the way it looks today. Z Gastroenterol. 2016;54(10):1156-1165.

3. Almaramhy HH. Acute appendicitis in young children less than 5 years: review article. Ital J Pediatr. 2017;43(1):15.

4. Shogilev DJ, Duus N, Odom SR, Shapiro NI. Diagnosing appendicitis: evidence-based review of the diagnostic approach in 2014. West JEmerg Med. 2014;15(7):859-871.

5. Doria AS, Moineddin R, Kellenberger CJ, et al. US or CT for diagnosis of appendicitis in children and adults? A meta-analysis. Radiology. 2006;241(1):83-94.

6. Brenner DJ, Hall EJ. Computed tomography - an increasing source of radiation exposure. $N$ Engl J Med. 2007;357(22):2277-2284.

7. Berrington de González A, Mahesh M, Kim KP, et al. Projected cancer risks from computed tomographic scans performed in the United States in 2007. Arch Intern Med. 2009;169(22):2071-2077.

8. Patino M, Fuentes JM, Singh S, Hahn PF, Sahani DV. Iterative reconstruction techniques in abdominopelvic $\mathrm{CT}$ : technical concepts and clinical implementation. AJR Am J Roentgenol. 2015;205(1): W19-W31.

9. Samuel M. Pediatric appendicitis score. J Pediatr Surg. 2002;37(6): 877-881.

10. Poortman P, Oostvogel HJ, Bosma E, et al. Improving diagnosis of acute appendicitis: results of a diagnostic pathway with standard use of ultrasonography followed by selective use of CT. J Am Coll Surg. 2009;208(3):434-441.

11. Kim K, Kim YH, Kim SY, et al. Low-dose abdominal CT for evaluating suspected appendicitis. N Engl J Med. 2012;366(17):1596-1605.

12. Seo H, Lee KH, Kim HJ, et al. Diagnosis of acute appendicitis with sliding slab ray-sum interpretation of low-dose unenhanced CT and standard-dose i.v. contrast-enhanced CT scans. AJR Am J Roentgenol. 2009;193(1):96-105.

13. Ahn S; LOCAT group. LOCAT (low-dose computed tomography for appendicitis trial) comparing clinical outcomes following low- vs standard-dose computed tomography as the first-line imaging test in adolescents and young adults with suspected acute appendicitis: study protocol for a randomized controlled trial. Trials. 2014;15:28.
14. Yi DY, Lee KH, Park SB, et al. Accuracy of low dose CT in the diagnosis of appendicitis in childhood and comparison with USG and standard dose CT. J Pediatr (Rio J). Epub 2017 April 23.

15. Ramarajan N, Krishnamoorthi R, Barth R, et al. An interdisciplinary initiative to reduce radiation exposure: evaluation of appendicitis in a pediatric emergency department with clinical assessment supported by a staged ultrasound and computed tomography pathway. Acad Emerg Med. 2009;16(11):1258-1265.

16. Thirumoorthi AS, Fefferman NR, Ginsburg HB, Kuenzler KA, Tomita SS. Managing radiation exposure in children - reexamining the role of ultrasound in the diagnosis of appendicitis. J Pediatr Surg. 2012;47(12):2268-2272.

17. Kotagal M, Richards MK, Flum DR, Acierno SP, Weinsheimer RL, Goldin AB. Use and accuracy of diagnostic imaging in the evaluation of pediatric appendicitis. J Pediatr Surg. 2015;50(4):642-646.

18. Kotagal M, Richards MK, Chapman T, et al; Safe and Sound Campaign. Improving ultrasound quality to reduce computed tomography use in pediatric appendicitis: the Safe and Sound campaign. Am J Surg. 2015;209(5):896-900.

19. Bachur RG, Hennelly K, Callahan MJ, Monuteaux MC. Advanced radiologic imaging for pediatric appendicitis, 2005-2009: trends and outcomes. J Pediatr. 2012;160(6):1034-1038.

20. Macco S, Vrouenraets BC, de Castro SM. Evaluation of scoring systems in predicting acute appendicitis in children. Surgery. 2016;160(6): 1599-1604.

21. Lourenco P, Brown J, Leipsic J, Hague C. The current utility of ultrasound in the diagnosis of acute appendicitis. Clin Imaging. 2016;40(5): 944-948.

22. Goldman RD, Carter S, Stephens D, Antoon R, Mounstephen W, Langer JC. Prospective validation of the pediatric appendicitis score. J Pediatr. 2008;153(2):278-282.

23. Zúñiga RV, Arribas JL, Montes SP, et al. Application of pediatric appendicitis score on the emergency department of a secondary level hospital. Pediatr Emerg Care. 2012;28(6):489-492.

24. Srinivasan A, Servaes S, Peña A, Darge K. Utility of CT after sonography for suspected appendicitis in children: integration of a clinical scoring system with a staged imaging protocol. Emerg Radiol. 2015; 22(1):31-42.

25. Rezak A, Abbas HM, Ajemian MS, Dudrick SJ, Kwasnik EM. Decreased use of computed tomography with a modified clinical scoring system in diagnosis of pediatric acute appendicitis. Arch Surg. 2011; 146(1):64-67.
Therapeutics and Clinical Risk Management

\section{Publish your work in this journal}

Therapeutics and Clinical Risk Management is an international, peerreviewed journal of clinical therapeutics and risk management, focusing on concise rapid reporting of clinical studies in all therapeutic areas, outcomes, safety, and programs for the effective, safe, and sustained use of medicines. This journal is indexed on PubMed Central, CAS,

\section{Dovepress}

EMBase, Scopus and the Elsevier Bibliographic databases. The manuscript management system is completely online and includes a very quick and fair peer-review system, which is all easy to use. Visit $\mathrm{http}: / /$ www.dovepress.com/testimonials.php to read real quotes from published authors. 